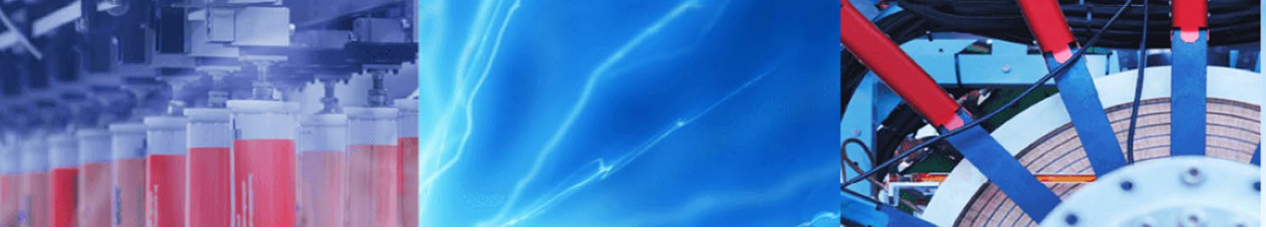

Research Article

\title{
Significances of prescribed heat sources on magneto Casson nanofluid flow due to unsteady bi-directionally stretchable surface in a porous medium
}

\author{
Muhammad Faisal $^{1}$ (D) $\cdot$ Iftikhar Ahmad' ${ }^{1}$ Tariq Javed $^{2}$
}

Received: 25 January 2020 / Accepted: 24 July 2020 / Published online: 4 August 2020

(c) Springer Nature Switzerland AG 2020

\begin{abstract}
In this communication, bidirectional flow of Casson nanomaterial driven by an unsteady moveable surface in the region of boundary layer is analyzed. Moreover, the significances of porous space, magnetic field, prescribed surface temperature (PST), and prescribed surface heat flux are also incorporated. Furthermore, the aspects of Brownian motion and thermophoresis are also comprised through Buongiorno nanofluid model. Governing equations are firstly transformed into system of ordinary differential equations by using a suitable combination of variables, and then computational assessment is made through Keller-Box method. Graphical illustrations for temperature distribution, concentration distribution, local Nusselt number and local Sherwood number against escalating amounts of pertinent parameters are presented. It is observed that escalating amounts of unsteady parameter and temperature controlled indices reduce the temperature distribution, as well as the concentration distribution. It is also observed that increasing amounts of Casson parameter enhances the rate of heat transfer, and reduces the rate of mass transfer for PST case. Finally, a comparison benchmark for limited case has been presented to validate the present methodology.
\end{abstract}

Keywords Casson nanofluid · Eckert number · Keller-Box method · MHD · Porous medium · Prescribed heat sources · Unsteady bi-directional stretching

\begin{tabular}{|c|c|c|c|}
\hline \multicolumn{2}{|c|}{ List of symbols } & $K$ & Permeability of porous medium $\left(\mathrm{m}^{2}\right)$ \\
\hline$a, b, c$ & Stretching rates $\left(\mathrm{s}^{-1}\right)$ & $k_{c}$ & Dynamic viscosity $\left(\mathrm{m}^{-1} \mathrm{~kg} \mathrm{~s}^{-1}\right)$ \\
\hline$B_{o}$ & Magnitude of magnetic force $\left(\mathrm{kg} \mathrm{s}^{-2} \mathrm{~A}^{-1}\right)$ & $M$ & Magnetic parameter $(-)$ \\
\hline$C$ & Concentration $\left(\mathrm{kg} \mathrm{m}^{-3}\right)$ & $N_{b}$ & Brownian motion parameter (-) \\
\hline$c_{f}$ & Specific heat of liquid $\left(\mathrm{m}^{2} \mathrm{~s}^{-2} \mathrm{~K}^{-1}\right)$ & $N_{t}$ & Thermophoresis parameter (-) \\
\hline$c_{p}$ & Specific heat of nanomaterial $\left(\mathrm{m}^{2} \mathrm{~s}^{-2} \mathrm{~K}^{-1}\right)$ & $N u_{x}$ & Local Nusslet number (-) \\
\hline$C_{\infty}^{p}$ & Ambient concentration $\left(\mathrm{kg} \mathrm{m}^{-3}\right)$ & $S h_{x}$ & Local Sherwood number (-) \\
\hline$C_{w}$ & Surface concentration $\left(\mathrm{kg} \mathrm{m}^{-3}\right)$ & $n_{p}$ & Number of grid points $(-)$ \\
\hline$C_{f x}, C_{f y}$ & Skin-friction coefficients (-) & $\operatorname{Pr}$ & Prandtl number (-) \\
\hline$D_{B}$ & Brownian coefficient $\left(\mathrm{m}^{2} \mathrm{~s}^{-1}\right)$ & $R e_{x}, R e_{y}$ & Reynolds numbers (-) \\
\hline$D_{T}$ & Thermophoresis coefficient $\left(\mathrm{m}^{2} \mathrm{~s}^{-1}\right)$ & $r, \hat{s}$ & Indices (-) \\
\hline$E c_{x}, E c_{y}$ & Eckert numbers (-) & $S$ & Unsteady parameter (-) \\
\hline$f, g$ & Similarity functions for velocity (-) & $T$ & Temperature $(\mathrm{K})$ \\
\hline$h_{j}$ & Step size for grids (m) & $T_{\infty}$ & Ambient temperature $(\mathrm{K})$ \\
\hline & Suffix (-) & $T_{w}$ & Surface temperature $(\mathrm{K})$ \\
\hline
\end{tabular}

Muhammad Faisal, muhammad.faisal@ajku.edu.pk| ${ }^{1}$ Department of Mathematics, Azad Jammu and Kashmir University, Muzaffarabad 13100, Pakistan. ${ }^{2}$ Department of Mathematics and Statistics, International Islamic University, Islamabad 44000, Pakistan. 


$\begin{array}{ll}t & \text { Time }(\mathrm{s}) \\ u, v, w & \text { Velocity components }\left(\mathrm{m} \mathrm{s}^{-1}\right) \\ x, y, z & \text { Space coordinates }(\mathrm{m})\end{array}$

\section{Greek letters}

$\alpha \quad$ Stretching ratio (-)

$\alpha_{m} \quad$ Thermal diffusivity $\left(\mathrm{m}^{2} \mathrm{~s}^{-1}\right)$

$\beta \quad$ Casson fluid parameter (-)

$\boldsymbol{\delta} \quad$ Unknown vector (-)

$\varepsilon \quad$ Porosity parameter (-)

$\eta \quad$ Similarity variable (-)

$\theta \quad$ Similarity function for temperature (-)

$\vartheta \quad$ Kinematic viscosity $\left(\mathrm{m}^{2} \mathrm{~s}^{-1}\right)$

$\lambda \quad$ Thermal conductivity $\left(\mathrm{m} \mathrm{kg} \mathrm{s}^{-3} \mathrm{~K}^{-1}\right)$

$\rho \quad$ Density of liquid $\left(\mathrm{m}^{-3} \mathrm{~kg}\right)$

$\sigma \quad$ Electrical conductivity $\left(\mathrm{m}^{-3} \mathrm{~kg}^{-1} \mathrm{~s}^{3} \mathrm{~A}^{2}\right)$

$\tau_{0} \quad$ Yield stress $\left(\mathrm{m}^{-1} \mathrm{~kg} \mathrm{~s}^{-2}\right)$

$\tau \quad$ Adiabatic index $\left(\mathrm{kg} \mathrm{m}^{-1}\right)$

$\phi \quad$ Similarity function for concentration (-)

\section{Introduction}

Stretching surface phenomenon is a widely used mechanism to analyze the fluid flow in the expanse of boundary layer, and it has intended many researchers owing to its multiplicity of usages in technological industries and engineering fields. Pioneer work of Crane [1] to obtain the series solution of the liquid flow caused by a moveable surface is settled the foundation to use this phenomenon for industrial and engineering purposes. This phenomenon has lot of applications like: fibrous insulations, construction of energy efficient buildings, solidification of casting, winding of electrical machinery, transpiration cooling, energy efficient dryers, ground water hydrology, grain storage installation, separation processes, parasitic losses, polymerase chain reaction, oil refining process and grinding, etc. Later on, hydromagnetic flow driven by a permeable stretchable surface has pulled the focus of various examiners due to its applications in polymerases technologies and metallurgy. Chakrabarti and Gupta [2] firstly examined the hydromagnetic flow over a stretchable surface to obtain the resemblance solution for velocity and heat transport characteristics through porous space. Analytical solution for generalized bidirectional hydromagnetic flow driven by a stretchable sheet saturated porous medium is obtained by Hayat and Javed [3]. The concept of fluid flow driven by a bi-directionally stretchable surface with prescribed heat mechanisms was introduced by Liu and Andersson [4], where heat transport characteristic for boundary layer flow is analyzed through similarity transformations. Elbashbeshy and Basid [5] firstly analyzed the heat transport mechanism for fluid flow over an unsteady stretchable surface. This type of study has been carried out through references [6-11] under the various aspects of fluid flow characteristics.

Hydromagnetic viscoelastic liquid flow driven by a bidirectionally moveable surface with heat transport characteristic in the expanse of boundary layer is numerically treated by Ahmad and Nazar [12] using Keller-Box method, and they concluded through this study that temperature rises for escalating amount of magnetic constraint. Now, in the classifications of non-Newtonian fluids, Casson liquid has distinctive structure because of its rheological identities corresponds to the shear stress-strain relations. It looks like an elastic solid at small shear strain, and beyond a critical stress amount, it behaves alike a Newtonian fluid. A Casson fluid can be best explained as a shear thinning liquid with zero viscosity at an infinite rate of shear, and infinite viscosity at a zero rate of shear. Most common examples having these type of characteristics are jelly, honey, tomato sauce, ketchup, jell, concentrated fruit juices, soup, chocolate, ice cream, and human blood, whereas industrial applications include molten plastics, slurries, and polymeric products. Nadeem et al. [13] has considered the hydromagnetic Casson fluid flow by reason of bi-directionally moveable surface in the expanse of boundary layer embedded porous medium, computationally by using shooting technique, and has deduced that flow velocity decreases for large amount of Casson material constraint. Spectral relaxation method is used by Mahanta and Shaw [14] for the investigation of Casson fluid flow because of bi-directional stretching porous surface with the influence of convective heat transport.

Recently, the investigation of nanofluid flow has achieved an incredible peak because of its lot of usages in chemical and pharmaceutical industries, heat transfer devices associated with condensers and boilers, cooling system of nuclear reactors and automobile engines, solar collectors and nanolubricants, macro plus micro-electronics, geothermal extraction and nano emulsification, improvement of power plants and thermal reservoirs, microelectromechanical systems, cooling of microchips, nanoliquid detergent, space technology, and many more. Buongiorno [15] illustrated a novel mathematical model to investigate the framework of nanofluid that describes the features of thermophoresis and Brownian movement. Khan and Pop [16] described the solution of nanomaterial flow over a moveable surface in the expanse of boundary layer, numerically, which was probably the first attempt on nanofluid flow over a moveable surface by using twophase nanoliquid model. Convective heat and mass transportations on magnetized Casson nanoliquid flow caused by a moveable surface was illustrated by Haq et al. [17], numerically. Nadeem et al. [18] deliberated the convective Casson nanofluid flow due to moving boundaries, 
and optimal solution of the modeled problem has been presented. Nadeem et al. [19] also discussed the hydromagnetic Casson nanofluid flow owing to bi-directionally moveable surface in the expanse of boundary layer with the stimulus of convective heat transport, numerically. Hayat et al. [20] addressed the mixed convective nanofluid flow of Casson liquid as a result of linearly stretchable surface in the boundary layer region under the influences of heat generation and chemical reaction, analytically. Hussain et al. [21] explored the series solution of Casson nanoliquid flow owing to linearly stretchable surface under viscous dissipation effect. More studies are carried out for Casson nanoliquid flow due to stretchable surface by many researchers [22-27], under the influences of various mechanisms like: Joule heating, porous medium, convective heat transport, hydromagnetic flow, slip conditions, thermal radiation, inclined surface, etc. Chamkha et al. [28] discussed the similarity solution for unsteady nanofluid flow because of moveable surface in the expanse of boundary layer, under the inspiration of chemical reaction through porous media with suction/injection parameters. Pushpalatha et al. [29] firstly described the unsteady boundary layer flow of Casson nanoliquid caused by a moveable surface under the impact of magnetic force with convectively heated wall, where series solution has been presented by using perturbation method.

In the sight of above literature, we tried to explore the unsteady boundary layer flow of Casson nanoliquid driven by a bi-directionally moveable surface through porous space with the effects of magnetic field and prescribed heat source, namely, PST and PHF. The novelty of this work is that a comprehensive study related to the Casson nanomaterial has been presented to check the influences of prescribed heat sources on rates of heat and mass transferences. The whole manuscript consists on the four major sections. In the first section, mathematical modeling of the physical model has been presented. In the second section, numerical solution has been obtained through Keller-Box method [30-37]. In the third section, graphical and tabular illustrations have been made to analyze the temperature distribution, concentration distribution, rate of heat transfer, and the rate of mass transfer, against wide ranges of involved parameters. In the last section, conclusion of the whole parametric study has been made and implication of the present work has been discussed.

\section{Modeling}

Consider the hydromagnetic Casson nanofluid flow owing to an unsteady bi-directional stretching surface in the region of boundary layer through porous media with the controls of prescribed heat mechanisms, namely, PST and
PHF. Frame of Cartesian coordinate is chosen for flow field analysis, where $x$ - and $y$-axes are supposed to be in the directions of stretching velocities, while $z$-axis is adjusted at right angle to the moveable surface, and fluid occupies the region $z>0$. Initially, at $t \leq 0$ both the fluid and the surface are kept at rest, and suddenly motion activates for $t>0$ with expanding velocities $U_{w}(x, t)=\frac{a x}{1-c t}$ along $x$-direction and $V_{w}(y, t)=\frac{b y}{1-c t}$ along $y$-direction. The special influences of magnetic field $\vec{B}$ and porous medium are incorporated through Lorentz force and Darcy's law, respectively, while the effects of induced and electric magnetic fields are ignored. Moreover, the influence of magnetic field is also incorporated into the heat equation, and the analysis of Casson nanofluid is conceded out using two-phase nanofluid model. Furthermore, the physical explanation of the current investigation is described through Fig. 1.

The rheological tensor $\tau_{i j}$ for an incompressible and isotropic flow of Casson liquid can be composed as: (see Refs. [7-9])

$\tau_{i j}= \begin{cases}2\left(k_{c}+\frac{\tau_{0}}{\sqrt{2 \pi}}\right) e_{i j} & \text { if } \pi>\pi_{c^{\prime}} \\ 2\left(k_{c}+\frac{\tau_{0}}{\sqrt{2 \pi_{c}}}\right) e_{i j} \text { if } \pi<\pi_{c^{\prime}}\end{cases}$

Here $e_{i j}$ symbolized for the $(i, j)$ th element of the deformation rate, $\pi$ symbolized for the multiplication of $e_{i j}$ with itself, $k_{c}$ symbolized for the dynamic viscosity of Casson liquid, $\tau_{0}$ symbolized for the yield-stress of the liquid, $\pi_{c}$ symbolized for the critical value of $\pi$ and $e_{i j}$ can be expressed as: (see Refs. $[13,14])$

$e_{i j}=\frac{1}{2}\left(\frac{\partial u_{i}}{\partial x_{j}}+\frac{\partial u_{j}}{\partial x_{i}}\right)$

The principal equations for mass, momentum, and energy conservations with nanoparticle concentration using above considerations can be composed as: (see Refs. $[22,29,30])$

$\frac{\partial u}{\partial x}+\frac{\partial v}{\partial y}+\frac{\partial w}{\partial z}=0$

$\frac{\partial u}{\partial t}+u \frac{\partial u}{\partial x}+v \frac{\partial u}{\partial y}+w \frac{\partial u}{\partial z}=\vartheta\left(1+\frac{\tau_{0}}{k_{c} \sqrt{2 \pi_{c}}}\right) \frac{\partial^{2} u}{\partial z^{2}}-\left(\frac{\vartheta}{K}+\frac{\sigma B_{0}^{2}}{\rho}\right) u$,

$\frac{\partial v}{\partial t}+u \frac{\partial v}{\partial x}+v \frac{\partial v}{\partial y}+w \frac{\partial v}{\partial z}=\vartheta\left(1+\frac{\tau_{0}}{k_{c} \sqrt{2 \pi_{c}}}\right) \frac{\partial^{2} v}{\partial z^{2}}-\left(\frac{\vartheta}{K}+\frac{\sigma B_{0}^{2}}{\rho}\right) v$, 
Fig. 1 Physical description of the present investigation

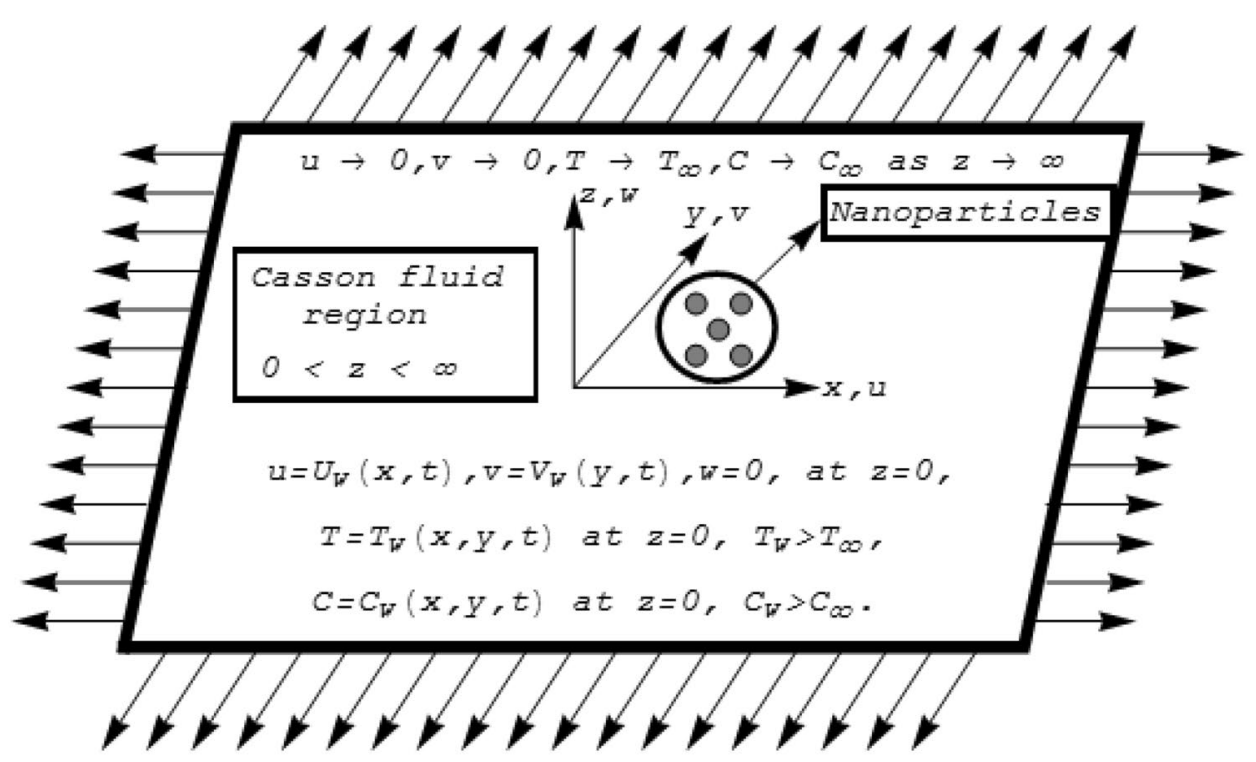

$\frac{\partial T}{\partial t}+u \frac{\partial T}{\partial x}+v \frac{\partial T}{\partial y}+w \frac{\partial T}{\partial z}=\alpha_{m} \frac{\partial^{2} T}{\partial z^{2}}+\tau\left[D_{B}\left(\frac{\partial C}{\partial z} \frac{\partial T}{\partial z}\right)+\frac{D_{T}}{T_{\infty}}\left(\frac{\partial T}{\partial z}\right)^{2}\right]+\frac{\sigma B_{0}^{2}}{(\rho C)_{f}}\left(u^{2}+v^{2}\right)$,

$\frac{\partial C}{\partial t}+u \frac{\partial C}{\partial x}+v \frac{\partial C}{\partial y}+w \frac{\partial C}{\partial z}=D_{B} \frac{\partial^{2} C}{\partial z^{2}}+\frac{D_{T}}{T_{\infty}} \frac{\partial^{2} T}{\partial z^{2}}$.

Here $t$ signifies the time, $(u, v, w)$ symbolize the velocity components along $(x-, y-, z-)$ directions, correspondingly, $T$ indicates the temperature, $C$ indicates the concentration, $\vartheta=\frac{k_{c}}{\rho}$ indicates the kinematic viscosity consists on the ratio form of dynamic viscosity $k_{c}$ with the density $\rho$ of fluid, $K$ represents the permeability of the porous space, $\sigma$ signifies the electric conductivity, $B_{0}$ represents the magnitude of magnetic field vector, $\alpha_{m}=\frac{\lambda}{(\rho c)_{f}}$ indicates the thermal diffusivity consists on the ratio form of thermal conductivity $\lambda$ with the volumetric heat capacity $(\rho c)_{f}$, $\tau=\frac{(\rho c)_{p}}{(\rho c)_{f}}$ denotes the adiabatic index consists on the ratio of thermal capacity of nanomaterial $(\rho c)_{p}$ with the thermal capacity of base liquid $(\rho c)_{f}, D_{B}$ describes the Brownian diffusion, while $D_{T}$ describes the thermophoresis diffusion, and $T_{\infty}$ represents the surrounding temperature. The typical boundary restrictions in the view of present continuation are (see Refs. [4, 31])

$u=\frac{a x}{1-c t}, v=\frac{b y}{1-c t}, w=0$ at $z=0, u \rightarrow 0, v \rightarrow 0$ as $z \rightarrow \infty$,
Here, $(a, b, c)$ are the stretching rates, $(r, s)$ represent the regulatory indices, $C_{\infty}$ represents the surrounding concentration, and $\left(T_{0}, C_{0}, T_{1}, C_{1}\right)$ are positive constants having intrinsic dimensions. Moreover, it is also deduced that Eqs. (8) and (9) are valid only for $t \leq \frac{1}{c}$ with $c \neq 0$. Now, we are introducing the following combination of variables to change the principal equations into dimensionless forms (see Refs. [32])

$u=\frac{a x}{1-c t} f^{\prime}(\eta), v=\frac{a y}{1-c t} g^{\prime}(\eta), w=-\sqrt{\frac{\vartheta a}{1-c t}}(f(\eta)+g(\eta)), \eta=\sqrt{\frac{a}{\vartheta(1-c t)}} z$,

PSTcase : $\quad \theta(\eta)=\frac{T-T_{\infty}}{T_{w}-T_{\infty}}, \phi(\eta)=\frac{C-C_{\infty}}{C_{w}-C_{\infty}}$,

PHFcase : $\quad T-T_{\infty}=\frac{T_{1}}{\lambda} \sqrt{\frac{9}{a(1-c t)}} x^{r} y^{s} \theta(\eta), C-C_{\infty}=\frac{C_{1}}{D_{B}} \sqrt{\frac{\vartheta}{a(1-c t)}} x^{r} y^{s} \phi(\eta)$.

Here $f, g, \theta$ and $\phi$ are the similarity functions for stretching velocity along $x$-direction, stretching velocity along $y$-direction, temperature distribution and concentration distribution, respectively, while $\eta$ is the independent similarity variable. It is noted that the Eq. (3) is straightforwardly fulfilled and remaining Eqs. (4)-(7) are transformed under the influence of Eq. (10) into the following forms:

$\left(1+\frac{1}{\beta}\right) f^{\prime \prime \prime}+(f+g) f^{\prime \prime}-f^{\prime 2}-S\left(f^{\prime}+\left(\frac{\eta}{2}\right) f^{\prime \prime}\right)-\left(\varepsilon+M^{2}\right) f^{\prime}=0$,

PST case : $\quad T=T_{\infty}+T_{0}\left(\frac{x^{r} y^{5}}{1-c t}\right), C=C_{\infty}+C_{0}\left(\frac{x^{r} y^{s}}{1-c t}\right)$ at $z=0, T \rightarrow T_{\infty}, C \rightarrow C_{\infty}$ as $z \rightarrow \infty$,

PHF case : $\quad-\lambda \frac{\partial T}{\partial z}=T_{1}\left(\frac{x^{r} y^{s}}{1-c t}\right),-D_{B} \frac{\partial C}{\partial z}=C_{1}\left(\frac{x^{r} y^{s}}{1-c t}\right)$ at $z=0, T \rightarrow T_{\infty}, C \rightarrow C_{\infty}$ as $z \rightarrow \infty$. 
$\left(1+\frac{1}{\beta}\right) g^{\prime \prime \prime}+(f+g) g^{\prime \prime}-g^{\prime 2}-S\left(g^{\prime}+\left(\frac{\eta}{2}\right) g^{\prime \prime}\right)-\left(\varepsilon+M^{2}\right) g^{\prime}=0$,

Above physical quantities in the non-dimensional forms can be expressed as:

$\frac{1}{P r} \theta^{\prime \prime}+(f+g) \theta^{\prime}-\left(r f^{\prime}+s g^{\prime}\right) \theta-S\left(\theta+\left(\frac{\eta}{2}\right) \theta^{\prime}\right)+N_{b} \theta^{\prime} \phi^{\prime}+N_{t} \theta^{\prime 2}+M^{2}\left(E c_{x} f^{2}+E c_{y} g^{\prime 2}\right)=0$,

$\phi^{\prime \prime}+\operatorname{PrLe}\left((f+g) \phi^{\prime}-\left(r f^{\prime}+s g^{\prime}\right) \phi-S\left(\phi+\left(\frac{\eta}{2}\right) \phi^{\prime}\right)\right)+\frac{N_{t}}{N_{b}} \theta^{\prime \prime}=0$,

$\sqrt{\operatorname{Re}_{x}} C_{f x}=\left(1+\frac{1}{\beta}\right) f^{\prime \prime}(0),\left(\frac{x}{y}\right) \sqrt{\operatorname{Re}_{x}} C_{f y}=\alpha\left(1+\frac{1}{\beta}\right) g^{\prime \prime}(0)$,

$f(0)=g(0)=0, f^{\prime}(\eta=0)=1, g^{\prime}(\eta=0)=\alpha, f^{\prime}(\eta \rightarrow \infty) \rightarrow 0, g^{\prime}(\eta \rightarrow \infty) \rightarrow 0$,

PST case : $\quad \theta(\eta=0)=1, \phi(\eta=0)=1, \theta(\eta \rightarrow \infty) \rightarrow 0, \phi(\eta \rightarrow \infty) \rightarrow 0$,

PHF case : $\quad \theta^{\prime}(\eta=0)=-1, \phi^{\prime}(\eta=0)=-1, \theta(\eta \rightarrow \infty) \rightarrow 0, \phi(\eta \rightarrow \infty) \rightarrow 0$.

Here, $\beta=\frac{k_{c} \sqrt{2 \pi_{c}}}{\tau_{0}}$ symbolizes the Casson fluid parameter, $S=\frac{c}{a}$ indicates the unsteady constraint consists on the ratio of stretching rates, $M^{2}=\frac{\sigma B_{0}^{2}}{a \rho}$ symbolizes the magnetic constraint, $\varepsilon=\frac{\vartheta}{a K}$ represents the porosity constraint, $\operatorname{Pr}=\frac{\vartheta}{\alpha_{m}}$ specifies the Prandtl number, $N_{b}=\frac{\tau D_{B}\left(C_{w}-C_{\infty}\right)}{\vartheta}$ specifies the Brownian movement constraint, $N_{t}=\frac{\tau D_{T}\left(T_{w}-T_{\infty}\right)}{\vartheta T_{\infty}}$ specifies the thermophoretic constraint, $E c_{X}=\frac{U_{w}^{2}}{c_{f}\left(T_{s}-T_{\infty}\right)}$ symbolized for the Eckert number along $x$-direction, $E c_{y}=\frac{V_{w}^{2}}{c_{f}\left(T_{s}-T_{\infty}\right)}$ symbolized for the Eckert number along $y$-direction, $L e=\frac{\alpha_{m}}{D_{B}}$ symbolizes the Lewis number, and $\alpha=\frac{b}{a}$ symbolizes the ratio parameter. The quantities of physical interest for present investigation are wall skinfriction $C_{f x}$ along $x$-direction stretching side, wall skin-friction $C_{f y}$ along $y$-direction stretching side, local Nusselt number $N u_{x}$ for the prediction of heat transport, and local Sherwood number $S h_{x}$ for the prediction of mass transport. Mathematically, these quantities can be expressed as: (see Refs. [18, 19, 32]):
PST case : $\quad N u_{x}=-\theta^{\prime}(0) \sqrt{R e_{x}}, S h_{x}=-\phi^{\prime}(0) \sqrt{R e_{x}}$,

$$
N u_{y}=-\theta^{\prime}(0) \sqrt{R e_{y}}, S h_{y}=-\phi^{\prime}(0) \sqrt{R e_{y}}
$$

PHF case : $\quad N u_{x}=\frac{1}{\theta(0)} \sqrt{R e_{x}}, S h_{x}=\frac{1}{\phi(0)} \sqrt{R e_{x}}$

$$
N u_{y}=\frac{1}{\theta(0)} \sqrt{R e_{y}}, S h_{y}=\frac{1}{\phi(0)} \sqrt{R e_{y}} .
$$

Here, $\left(R e_{x}=\frac{x U_{w}}{\vartheta}, R e_{y}=\frac{y V_{w}}{\alpha \vartheta}\right)$ represent the local Reynolds numbers.

\section{Keller-Box scheme}

The next step after modeling of the mathematical model is the choice of the suitable method to solve the modeled system. In this continuation, we preferred the Keller-Box method (KBM) introduced by Keller [37], which is implicit in nature, in order to solve the set of nonlinear ODEs. Equations (11)-(14) subject to the classical boundary conditions

$C_{f x}=\frac{\tau_{x z}}{\rho U_{w}^{2}}, C_{f y}=\frac{\tau_{y z}}{\rho V_{w}^{2}}, N u_{x}=\frac{x q_{s}}{\lambda\left(T_{w}-T_{\infty}\right)}, S h_{x}=\frac{x j_{s}}{D_{B}\left(C_{w}-C_{\infty}\right)}, N u_{y}=\frac{y q_{s}}{\lambda\left(T_{w}-T_{\infty}\right)}, S h_{y}=\frac{y j_{s}}{D_{B}\left(C_{w}-C_{\infty}\right)}$.

Here, $\tau_{x z}$ labels the local shear stress along $x$-direction, while $\tau_{y z}$ labels the local shear stress along $y$-direction, $q_{s}$ labels the local surface heats flux, while $j_{s}$ symbolizes the local surface mass flux and these are defined as:

$\tau_{x z}=\left(k_{c}+\frac{\tau_{0}}{\sqrt{2 \pi_{c}}}\right)\left(\frac{\partial u}{\partial z}\right)_{z=0^{\prime}} \quad \tau_{y z}=\left(k_{c}+\frac{\tau_{0}}{\sqrt{2 \pi_{c}}}\right)\left(\frac{\partial v}{\partial z}\right)_{z=0^{\prime}}$

$q_{s}=-\lambda\left(\frac{\partial T}{\partial z}\right)_{z=0} \quad j_{s}=-D_{B}\left(\frac{\partial C}{\partial z}\right)_{z=0}$.
Eq. (15), numerically. This method has second-order accuracy, and has a lot of advantages over other numerical approaches (SLM, shooting, BVP4C, RK, etc.). These advantages are listed below:

- It has faster convergence than other numerical approaches.

- Mixed boundary conditions can be easily tackled thorough Keller-Box approach.

- Accuracy of the solution can be easily achieved by reducing the step-size. 
- It is a more stable, more effectual, and more flexible to use than other numerical approaches.

The mathematical procedure to implement this numerical approach can be started by considering the new variables $H, G, P, Q, L$ and $W$ to alter the higher-order ODEs into the system of first-order ODEs as follows:

$f^{\prime}=H, H^{\prime}=G, g^{\prime}=P, P^{\prime}=Q, \theta^{\prime}=L, \phi^{\prime}=W$,

$\left(1+\frac{1}{\beta}\right) G^{\prime}+(f+g) G-H^{2}-S\left(H+\left(\frac{\eta}{2}\right) G\right)-\left(\varepsilon+M^{2}\right) H=0$,

$\left(1+\frac{1}{\beta}\right) Q^{\prime}+(f+g) Q-P^{2}-S\left(P+\left(\frac{\eta}{2}\right) Q\right)-\left(\varepsilon+M^{2}\right) P=0$,

$\frac{1}{P_{r}} L^{\prime}+(f+g) L-(r H+s P) \theta-S\left(\theta+\left(\frac{\eta}{2}\right) L\right)+N_{b} L W+N_{t} L^{2}$

$+M^{2}\left(E c_{x} H^{2}+E c_{y} P^{2}\right)=0$,

$W^{\prime}+\operatorname{PrLe}\left((f+g) W-(r H+s P) \phi-S\left(\phi+\left(\frac{\eta}{2}\right) W\right)\right)+\frac{N_{t}}{N_{b}} L=0$,
The rest of the procedure for Keller-Box method is completed through Newton's method and LU-decomposition process (Ref. [32]) to obtain the unknown vector $\delta$. Finally, desired accuracy of the solution is achieved through successive iterations by using in-house built code, and then the unknown vector $\delta$ is further used to obtain the values of physical quantities.

\section{Convergence}

The convergence of the solution of the Eqs. (11)-(15) is illustrated through Table 1 using grid independence test for different refinement levels $R_{\text {l }}$ against fixed and suitable amounts of involved constraints. For the first refinement

with the boundary restrictions

$f(0)=g(0)=0, H(\eta=0)=1, P(\eta=0)=\alpha, H(\eta \rightarrow \infty) \rightarrow 0, P(\eta \rightarrow \infty) \rightarrow 0$,

PST case : $\quad \theta(\eta=0)=1, \phi(\eta=0)=1, \theta(\eta \rightarrow \infty) \rightarrow 0, \phi(\eta \rightarrow \infty) \rightarrow 0$,

PHF case : $\quad L(\eta=0)=-1, W(\eta=0)=-1, \theta(\eta \rightarrow \infty) \rightarrow 0, \phi(\eta \rightarrow \infty) \rightarrow 0$.

The discretization scheme for Eq. (22) is expressed as:

$$
\begin{aligned}
& \eta_{0}=0, \eta_{j}=\eta_{j-1}+h_{j}, j=1,2,3, \ldots, J ; \eta_{j}=\eta_{\infty}, H_{j-\frac{1}{2}}=\frac{f_{j}-f_{j-1}}{h_{j}}, \\
& G_{j-\frac{1}{2}}=\frac{H_{j}-H_{j-1}}{h_{j}}, P_{j-\frac{1}{2}}=\frac{g_{j}-g_{j-1}}{h_{j}}, Q_{j-\frac{1}{2}}=\frac{P_{j}-P_{j-1}}{h_{j}}, L_{j-\frac{1}{2}}=\frac{\theta_{j}-\theta_{j-1}}{h_{j}}, W_{j-\frac{1}{2}}=\frac{\phi_{j}-\phi_{j-1}}{h_{j}}, \\
& \left(1+\frac{1}{\beta}\right) \frac{G_{j}-G_{j-1}}{h_{j}}+\left(f_{j-\frac{1}{2}}+g_{j-\frac{1}{2}}\right) G_{j-\frac{1}{2}}-H_{j-\frac{1}{2}}^{2}-S\left(H_{j-\frac{1}{2}}+\frac{\eta}{2} G_{j-\frac{1}{2}}\right)-\left(\varepsilon+M^{2}\right) H_{j-\frac{1}{2}}=0, \\
& \left(1+\frac{1}{\beta}\right) \frac{Q_{j}-Q_{j-1}}{h_{j}}+\left(f_{j-\frac{1}{2}}+g_{j-\frac{1}{2}}\right) Q_{j-\frac{1}{2}}-P_{j-\frac{1}{2}}^{2}-S\left(P_{j-\frac{1}{2}}+\frac{\eta}{2} Q_{j-\frac{1}{2}}\right)-\left(\varepsilon+M^{2}\right) P_{j-\frac{1}{2}}=0, \\
& \frac{1}{P r}\left(\frac{L_{j}-L_{j-1}}{h_{j}}\right)+(f+g) L_{j-\frac{1}{2}}-\left(r H_{j-\frac{1}{2}}+s P_{j-\frac{1}{2}}\right) \theta_{j-\frac{1}{2}}-S\left(\theta_{j-\frac{1}{2}}+\frac{\eta}{2} L_{j-\frac{1}{2}}\right)+N_{b} L_{j-\frac{1}{2}} W_{j-\frac{1}{2}} \\
& \quad+N_{t} L_{j-\frac{1}{2}}^{2}+M^{2}\left(E c_{x} H_{j-\frac{1}{2}}^{2}+E c_{y} P_{j-\frac{1}{2}}^{2}\right)=0, \\
& \frac{W_{j}-W_{j-1}}{h_{j}}+\operatorname{PrLe}\left(\left(f_{j-\frac{1}{2}}+g_{j-\frac{1}{2}}\right) W_{j-\frac{1}{2}}-\left(r H_{j-\frac{1}{2}}+s P_{j-\frac{1}{2}}\right) \phi_{j-\frac{1}{2}}-S\left(\phi_{j-\frac{1}{2}}+\frac{\eta}{2} W_{j-\frac{1}{2}}\right)\right) \\
& \quad+\left(\frac{N_{t}}{N_{b}}\right) \frac{L_{j}-L_{j-1}}{h_{j}}=0 .
\end{aligned}
$$

\begin{tabular}{|c|c|c|c|c|c|c|c|}
\hline \multirow[t]{2}{*}{$R_{l}$} & \multirow[t]{2}{*}{$n_{p}$} & \multirow[t]{2}{*}{$-f^{\prime \prime}(0)$} & \multirow[t]{2}{*}{$-g^{\prime \prime}(0)$} & \multicolumn{2}{|l|}{ PST case } & \multicolumn{2}{|l|}{ PHF case } \\
\hline & & & & $-\theta^{\prime}(0)$ & $-\phi^{\prime}(0)$ & $\overline{\theta(0)}$ & $\phi(0)$ \\
\hline $1 \mathrm{st}$ & 500 & 1.070235 & 0.494328 & 1.083489 & 1.266369 & 0.880629 & 0.834445 \\
\hline 2nd & 1000 & 1.070235 & 0.494325 & 1.083451 & 1.266414 & 0.880657 & 0.834429 \\
\hline $3 r d$ & 1500 & 1.070236 & 0.494325 & 1.083444 & 1.266423 & 0.880662 & 0.834426 \\
\hline 4th & 2000 & 1.070236 & 0.494324 & 1.083441 & 1.266426 & 0.880664 & 0.834425 \\
\hline 5 th & 2500 & 1.070236 & 0.494324 & 1.08344 & 1.266427 & 0.880665 & 0.834425 \\
\hline 6th & 3000 & 1.070236 & 0.494324 & 1.083439 & 1.266428 & 0.880665 & 0.834425 \\
\hline 7th & 3500 & 1.070236 & 0.494324 & 1.083439 & 1.266428 & 0.880665 & 0.834425 \\
\hline
\end{tabular}

Table 1 Solution of present model for different refinement levels when $\operatorname{Pr}=L e=1.2, S=\varepsilon=M$ $=N_{t}=N_{b}=\alpha=0.5$, $\beta=r=s=1.0, E c_{x}=E c_{y}=0.3$ 
level, the number of elements $n_{p}$ for the computed domain is chosen five-hundred, whereas for the second refinement level the number of elements is increased up-to one-thousand, and so on. The tolerance level of the convergence is adjusted to $10^{-6}$. It is noted that by increasing the number of elements, error in the numerical solution is reducing. The error in the computation is approaches to $0 \%$ for fourth refinement level. Hence, through-out this analysis, third refinement level is adopted to predict the amounts of heat and mass transports with $h_{j}=\frac{\eta_{\jmath}-\eta_{0}}{n_{p}}: \eta_{0}=0, \eta_{\jmath}=20$.

\section{Validation}

In order to ensure the accuracy of the applied methodology, the outcomes obtained through Keller-Box method are compared with the previously published results of "Nadeem et al. [13]" through Table 2. It is concluded through Table 2 that the two set of results for skin-friction coefficients are best matched with each other for limited steady state $(S=0)$ case.

\section{Results and discussion}

In this portion, the impact of embryonic involved constraints on temperature distribution, concentration distribution, local Nusselt number (for heat flux), and local Sherwood number (for mass flux) are briefly analyzed through various graphical illustrations. Special focus has been given to PST and PHF mechanisms.

\subsection{Temperature and concentration distributions}

The impacts of emerging involved constraints, namely, unsteady constraint $S$ and temperature controlled indices ( $r$ and $s$ ) on temperature $\theta(\eta)$ and concentration $\phi(\eta)$ distributions are analyzed in this section through Figs. 2, 3 and 4.

Figure 2a-d illustrate the impact of unsteady constraint $S$ on temperature distribution $\theta(\eta)$ for PST case, temperature distribution $\theta(\eta)$ for PHF case, concentration distribution $\phi(\eta)$ for PST case, and concentration distribution $\phi(\eta)$ for PHF case, respectively. It is observed through Fig. 2a-d that the escalating amount of unsteady constraint $S$ reduces the temperature $\theta(\eta)$ as well as concentration $\phi(\eta)$ distributions, for both PST and PHF mechanism. Moreover, temperature of the surface through Fig. $2 a-b$ is detected higher for PST source as compared to PHF source against augmenting amounts of $S$, whereas the thickness of thermal layer is noticed higher for PHF case as compared to PST case. Physically, escalating amount of $S$ increases the electric conductivity of the liquid and this increment in the electric conductivity is higher for PHF case as compared to PST case. As a result, temperature distribution is reduced against higher amounts of $S$. Furthermore, concentration distribution nearer to the surface through Fig. $2 c-d$ is observed higher for PST case than PHF case against higher amounts of $S$, whereas the thickness of concentration
Table 2 Comparison of skinfriction coefficient's values for a suitable ranges of $M, \beta, \alpha$ and $\varepsilon$ with previous data of "Nadeem et al. [13] "when $S=0$

\begin{tabular}{|c|c|c|c|c|c|c|}
\hline \multirow{3}{*}{$M$} & \multirow{3}{*}{$\beta$} & \multirow{3}{*}{$\varepsilon$} & \multicolumn{2}{|c|}{$\alpha=0$} & \multicolumn{2}{|c|}{$\alpha=1.0$} \\
\hline & & & \multicolumn{2}{|c|}{$-\left(1+\frac{1}{\beta}\right) f^{\prime \prime}(0)$} & \multicolumn{2}{|c|}{$-\left(1+\frac{1}{\beta}\right) g^{\prime \prime}(0)$} \\
\hline & & & Present & $\begin{array}{l}\text { "Nadeem et al. } \\
\text { [13] " }\end{array}$ & Present & $\begin{array}{l}\text { "Nadeem } \\
\text { et al. [13]" }\end{array}$ \\
\hline \multirow[t]{5}{*}{0.0} & $\infty$ & 0.0 & 1.00000 & 1.0042 & 1.17372 & 1.1748 \\
\hline & 5 & & 1.09545 & 1.0954 & 1.28575 & 1.2857 \\
\hline & 1 & & 1.41421 & 1.4142 & 1.65989 & 1.6599 \\
\hline & & 0.5 & 1.73205 & 1.7320 & 1.93156 & 1.9315 \\
\hline & & 1.0 & 2.00000 & 2.0000 & 2.17183 & 2.1718 \\
\hline \multirow[t]{5}{*}{10} & $\infty$ & 0.0 & 3.31662 & 3.3165 & 3.36726 & 3.3667 \\
\hline & 5 & & 3.63318 & 3.6331 & 3.68864 & 3.6886 \\
\hline & 1 & & 4.69042 & 4.6904 & 4.76201 & 4.7620 \\
\hline & & 0.5 & 4.79583 & 4.7958 & 4.86583 & 4.8658 \\
\hline & & 1.0 & 4.89898 & 4.8990 & 4.96749 & 4.9674 \\
\hline \multirow[t]{5}{*}{100} & $\infty$ & 0.0 & 10.0499 & 10.049 & 10.0665 & 10.066 \\
\hline & 5 & & 11.00909 & 11.0091 & 11.0273 & 11.0272 \\
\hline & 1 & & 14.21267 & 14.2127 & 14.2362 & 14.2361 \\
\hline & & 0.5 & 14.24781 & 14.2478 & 14.2713 & 14.2712 \\
\hline & & 1.0 & 14.28286 & 14.2829 & 14.3062 & 14.3062 \\
\hline
\end{tabular}



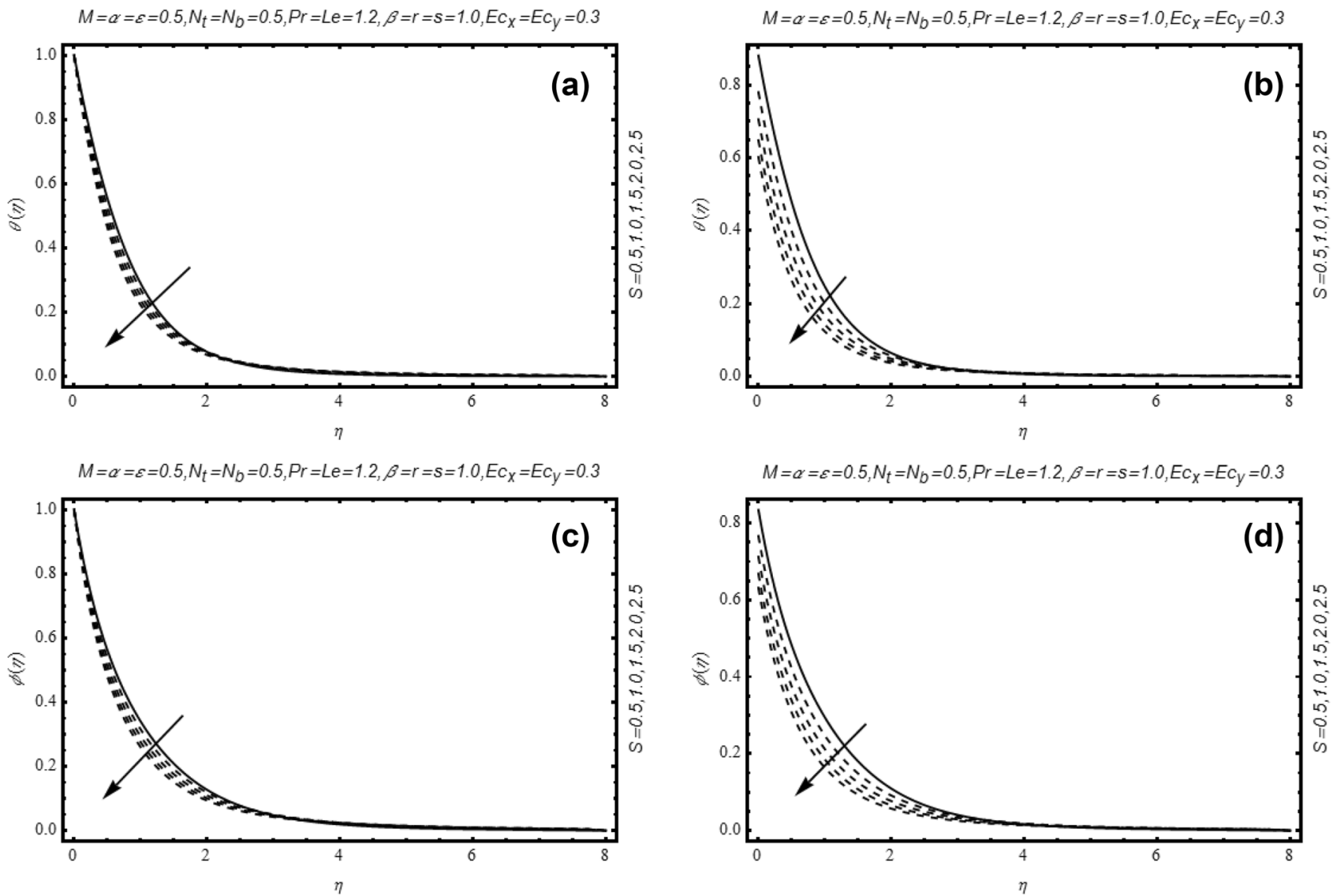

Fig. 2 Impact of unsteady constraint $S$ on temperature distribution $\theta(\eta)$ for [PST case (a) and PHF case (b)] and on concentration distribution $\phi(\eta)$ for [PST case $(\mathbf{c})$ and PHF case $(\mathbf{d})]$

layer is achieved higher for PHF case as compared to PST case. Mathematically, increasing amount of $S$ reduces the amount of thermal diffusivity and increases the amount of Brownian coefficient, as a result, concentration distribution is diminished against higher amounts of $S$.

Figure $3 a-d$ describe the impact of index $r$ on temperature distribution (PST case), temperature distribution (PHF case), concentration distribution (PST case), and concentration distribution (PHF case), respectively. It is noted through Fig. 3a-d that higher amount of $r$ diminishes the concentration $\phi(\eta)$ distribution as well as temperature $\theta(\eta)$ distribution for both the prescribed heat sources (PST and PHF). It is perceived through Fig. 3a-b that PHF source produces higher temperature $\theta(\eta)$ distribution than PST source for negative amounts of $r$, whereas opposite trend is achieved for positive amounts of $r$. Physically, positive amounts of $r$ enhance the wall temperature $T_{S}$ whereas negative amounts of $r$ diminish the wall temperature $T_{S}$, as a result, temperature distribution $\theta(\eta)$ is higher for negative amounts of $r$ than positive amounts of $r$. Similar trends are observed through Fig. $3 c-d$ for concentration $\phi(\eta)$ distribution against augmenting amounts of $r$ under PST and PHF mechanisms.

Figure 4a-d elucidate the influence of index $s$ on temperature distribution (PST case), temperature distribution (PHF case), concentration distribution (PST case), and concentration distribution (PHF case), respectively. It is deduced through Fig. $4 a-d$ that developed amount of $r$ lessens the concentration $\phi(\eta)$ distribution as well as temperature $\theta(\eta)$ distribution for both the prescribed heat sources (PST and PHF). It is perceived through Fig. $4 a-b$ that PHF source produces higher temperature $\theta(\eta)$ distribution than PST source for negative amounts of $s$, however reverse trend is achieved for positive amounts of s. Actually, positive amounts of $s$ improve the wall temperature $T_{S}$ while negative amounts of $s$ lessen the wall temperature $T_{s}$, as a result, temperature distribution $\theta(\eta)$ is enhanced for negative amounts of $r$ than positive amounts of $r$. Similar trends are observed through Fig. $4 \mathrm{c}-\mathrm{d}$ for concentration $\phi(\eta)$ distribution besides extending amounts of $s$ under PST and PHF mechanisms. 

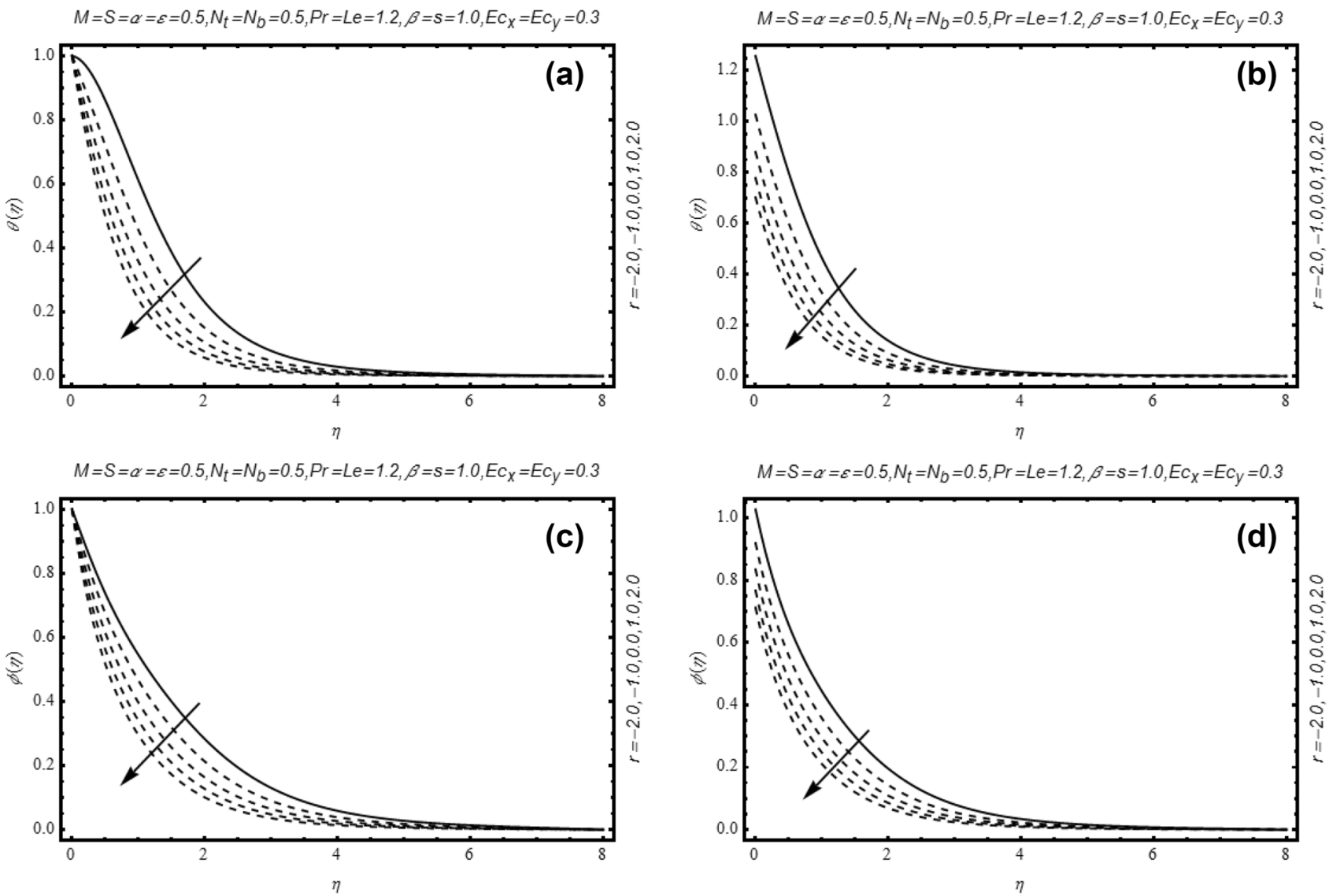

Fig. 3 Impact of temperature controlled index $r$ on temperature distribution $\theta(\eta)$ for [PST case (a) and PHF case (b)] and on concentration distribution $\phi(\eta)$ for [PST case $(\mathbf{c})$ and PHF case (d)]

\subsection{Heat and mass transports}

The present section describes the influences of temperature controlled indices ( $r$ and $s$ ), Brownian constraint $N_{b}$, and thermophoresis constraint $N_{t}$ on the rate of heat transfer for PST case $\theta^{\prime}(0)$, the rate of heat transfer for PHF case $\frac{1}{\theta(0)}$, the rate of mass transfer for PST case $\phi^{\prime}(0)$, and the rate of mass transfer for PHF case $\frac{1}{\phi(0)}$ through Figs. $5 \mathrm{a}$ and $6 \mathrm{~d}$. Moreover, tabular comparisons are also presented between the rates of heat transferences (for PST and PHF cases) through Table 3 and between the rates of mass transferences (for PST and PHF cases) through Table 4 against escalating amounts of magnetic constraint $M$, Eckert numbers $\left(E c_{x}\right.$ and $\left.E c_{y}\right)$, Prandtl number Pr, Casson parameter $\beta$, Lewis number $L e$, and unsteady constraint $S$.

Figure 5a illustrates the impacts of $N_{t}$ and $r$ on the local Nusslet number for PST case and it is deduced through this figure that increasing amounts of $N_{t}$ decline the rate of heat transfer, whereas escalating amounts of $r$ enhance the rate of heat transfer. Basically, higher amount of $N_{t}$ augments the thermal diffusivity of the liquid, as a result, rate of heat transfer is reduced. Similar trend is achieved for the local Nusslet number for PHF case through Fig. 5b, but the rate of heat transfer is higher for PHF case as compared to PST case. Figure $5 c$ describes the influences of $r$ and $N_{t}$ on the local Sherwood number (PST case) and it is extracted through this figure that growing amounts of $N_{t}$ also decline the amount of mass transport whereas growing amounts of $r$ progress the rate of mass transport. Mathematically, higher amount of $r$ reduces the concentration distribution $\phi(\eta)$, as a result, rate of mass flux is augmented for higher amounts of $r$. Identical tendency is attained for the local Sherwood number for PHF case through Fig. $5 \mathrm{~d}$ against escalating amounts of $r$ and $N_{t}$. It is observed through Fig. $5 \mathrm{c}-\mathrm{d}$ that the rate of mass flux is higher for PHF case as compared to PST case against growing amounts of $r$ and $N_{t}$.

Figure 6a demonstrates the effects of $N_{b}$ and $s$ on the local Nusslet number for PST situation and it is inferred through this figure that growing amounts of $N_{b}$ decay 

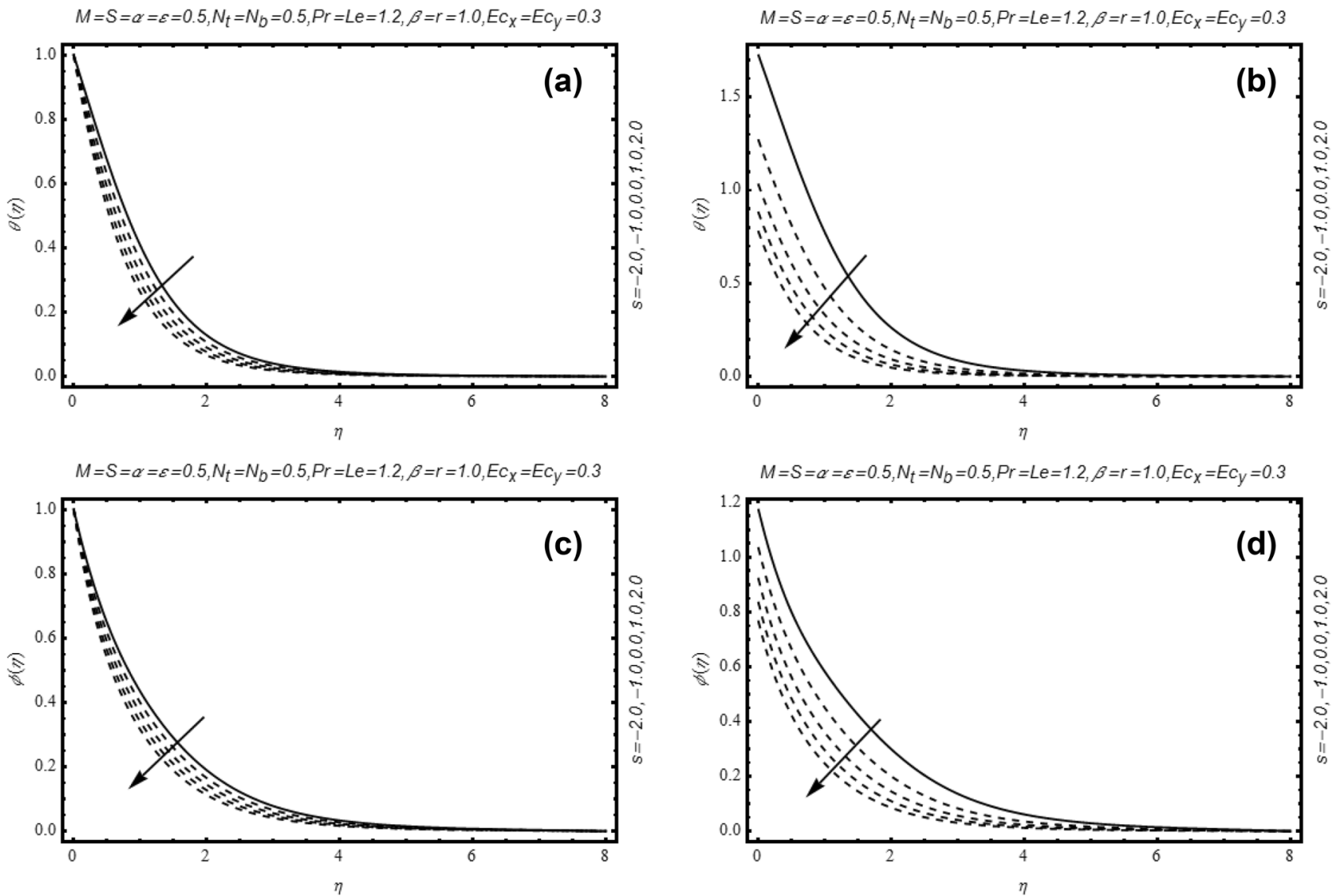

Fig. 4 Impact of temperature controlled index $s$ on temperature distribution $\theta(\eta)$ for [PST case (a) and PHF case (b)] and on concentration distribution $\phi(\eta)$ for [PST case $(\mathbf{c})$ and PHF case $(\mathbf{d})$ ]

the level of heat transport, whereas rising amounts of $s$ enrich the level of heat transport. Ultimately, developed amount of $N_{b}$ reduces the coefficient of Brownian diffusion, as a result, level of heat transport is decayed. Similar trend is seen for the level of heat transport for PHF case through Fig. $6 \mathrm{~b}$, but the level of heat transport is higher for PHF case as compared to PST case. Figure $6 \mathrm{c}$ pronounces the effects of $s$ and $N_{b}$ on the local Sherwood number (PST case) and it is noted through this figure that developing amounts of $N_{b}$ and senhance the level of mass transport. Mathematically, higher amount of $s$ diminishes the concentration distribution $\phi(\eta)$, as a result, level of mass flux is increased for higher amounts of $s$. Identical tendencies are attained for the local Sherwood number for PHF case through Fig. 6d against escalating amounts of $s$ and $N_{b}$. It is detected through Fig. $6 c$, $d$ that the level of mass flux is greater for PHF case as compared to PST case against mounting amounts of $s$ and $N_{b}$.
Table 3 elaborates the numerical comparison between the level of heat transfer for PST case and the level of heat transfer for PHF case. It is illustrated through Table 4 that growing amounts of magnetic constraint $M$, Lewis number $L e$, and Eckert number $\left(E c_{x}\right.$ and $\left.E c_{y}\right)$ reduce the level of heat transport whereas enlarging amounts of Prandtl number $P r$, Casson parameter $\beta$, and unsteady constraint $S$ enhance the level of heat transport. It is also deduced through Table 3 that PHF source produces the better rate of heat transfer as compared to PST source due to the negative influence of thermal conductivity in the PHF mechanism.

Table 4 illustrates the computational comparison between the level of mass transfer for PST case and the level of mass transfer for PHF case. It is detected through Table 4 that growing amounts of magnetic constraint $M$ and Casson parameter $\beta$ reduce the level of mass transport whereas expanding amounts of Eckert numbers $\left(E c_{x}\right.$ and $\left.E c_{y}\right)$, unsteady constraint $S$, Prandtl number Pr, 

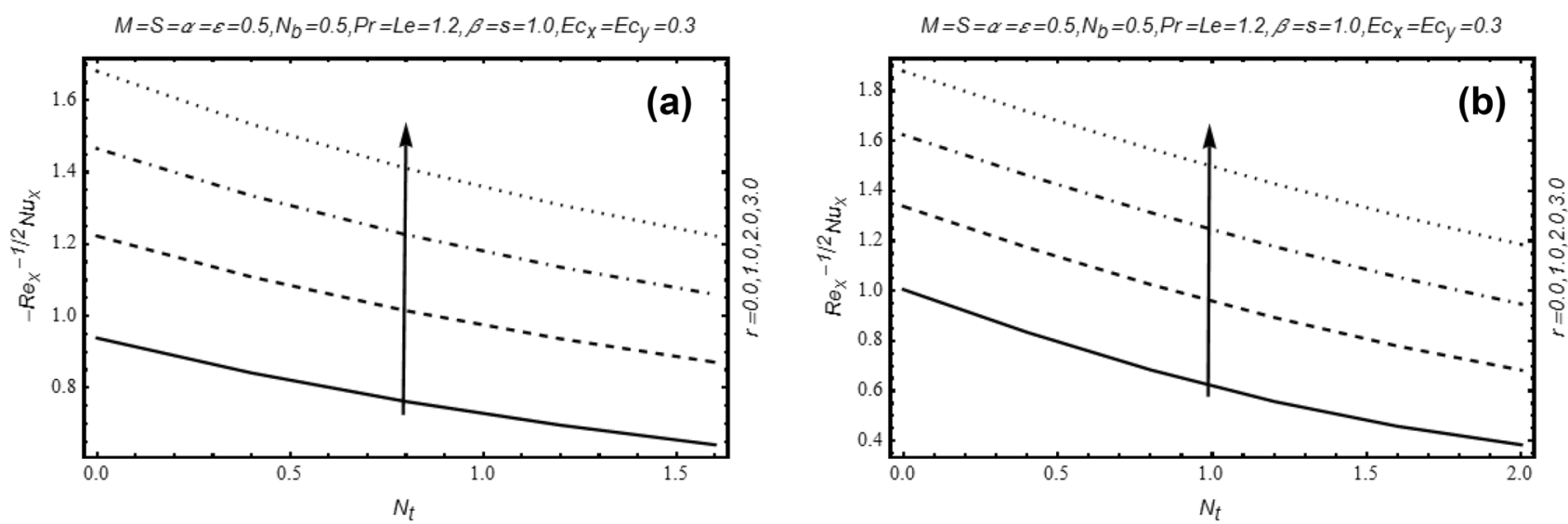

$M=S=\alpha=\varepsilon=0.5, N_{D}=0.5, \operatorname{Pr}=L e=1.2, \beta=S=1.0, E c_{X}=E c_{y}=0.3$
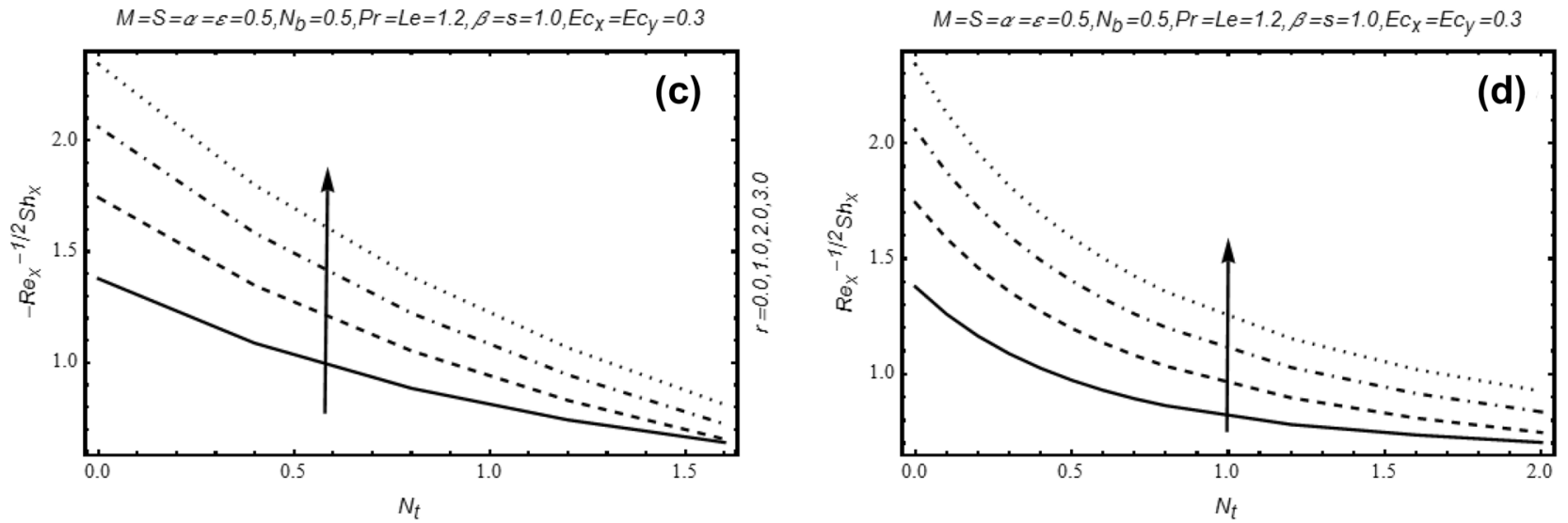

Fig. 5 Trends of local Nusselt number for [PST case (a) and for PHF case (b)] and local Sherwood number for [PST case (c) and PHF case (d)] against wide ranges of $N_{t}$ and $r$

and Lewis number Le enhance the level of mass transport. It is also deduced through Table 4 that PST source produces the better rate of mass transfer as compared to PHF source due to the negative influence of mass diffusivity in the PHF mechanism. 

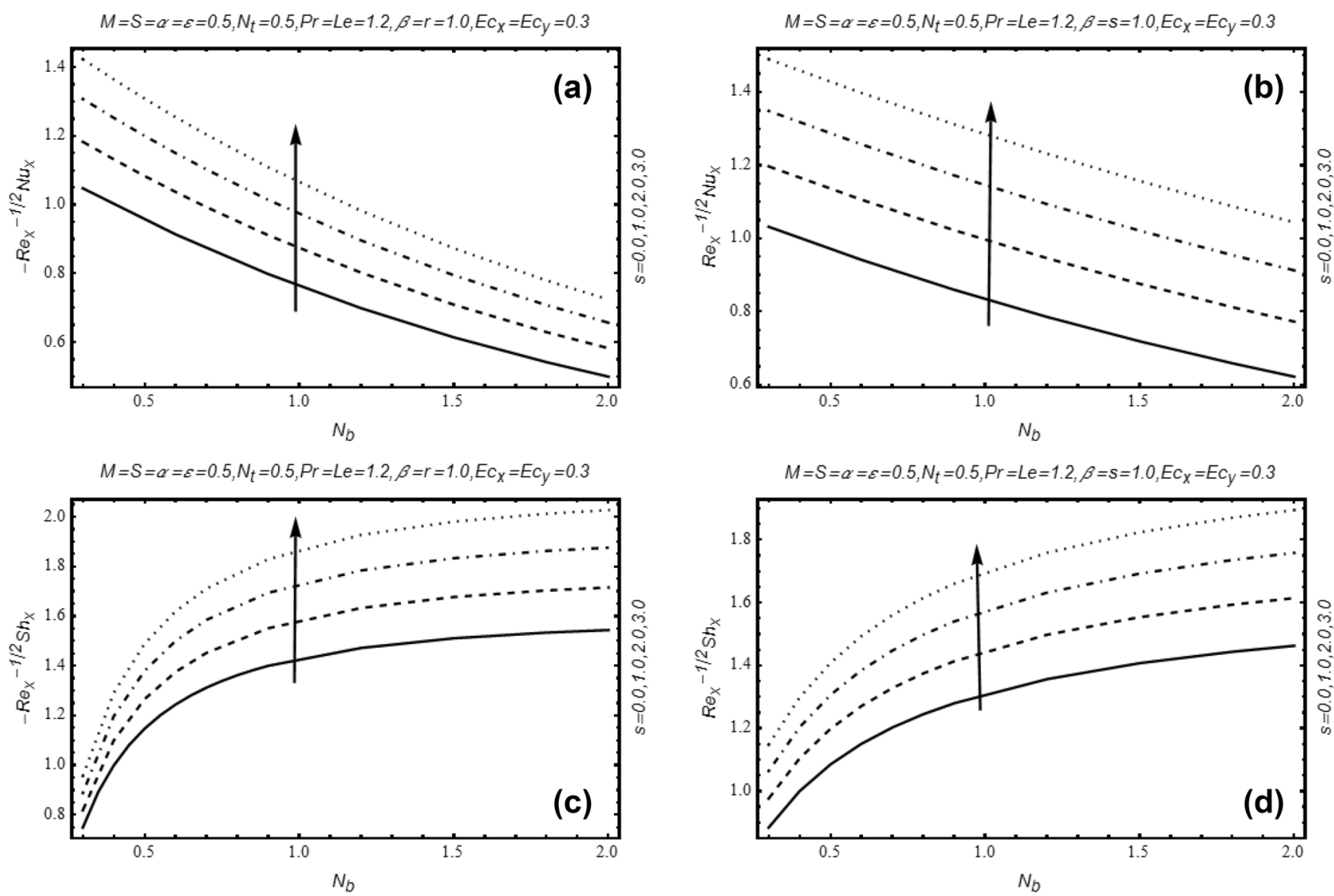

Fig. 6 Trends of local Nusselt number for [PST case (a) and for PHF case (b)] and local Sherwood number for [PST case (c) and PHF case (d)] against wide ranges of $N_{b}$ and $s$ 
Table 3 Trend of local

Nusselt number for PST and

PHF mechanisms against

escalating amounts of

magnetic constraint $M$, Prandtl

number $P r$, Lewis number

$L e$, Eckert numbers $\left(E c_{x}, E c_{y}\right)$,

Casson parameter $\beta$ and

unsteady constraint $S$ when

$\varepsilon=\alpha=0.3, N_{t}=N_{b}=0.7, r=s=1.0$

Table 4 Trend of local

Sherwood number for PST

and PHF mechanisms against

escalating amounts of

magnetic constraint $M$, Prandtl

number $P r$, Lewis number

Le, Eckert numbers $\left(E c_{x}, E c_{y}\right)$,

Casson parameter $\beta$ and

unsteady constraint $S$ when

$\varepsilon=\alpha=0.3, N_{t}=N_{b}=0.7, r=s=1.0$

\begin{tabular}{|c|c|c|c|c|c|c|c|c|c|}
\hline \multirow{2}{*}{$M$} & \multirow{2}{*}{$\operatorname{Pr}$} & \multirow{2}{*}{ Le } & \multirow{2}{*}{$E c_{x}$} & \multirow{2}{*}{$E c_{y}$} & \multirow{2}{*}{$\beta$} & \multirow{2}{*}{$S$} & \multicolumn{3}{|c|}{$\frac{N u_{x}}{\sqrt{R e_{x}}}=\frac{N u_{y}}{\sqrt{R e_{y}}}$} \\
\hline & & & & & & & PST case $\left[-\theta^{\prime}(0)\right]$ & PHF case & {$\left[\frac{1}{\theta(0)}\right]$} \\
\hline 0.5 & 1.2 & 1.2 & 0.1 & 0.1 & 1.0 & 0.5 & 0.917115 & 0.948989 & \\
\hline 1.0 & - & - & - & - & - & - & 0.884277 & 0.904043 & \\
\hline 1.5 & - & - & - & - & - & - & 0.837420 & 0.846168 & \\
\hline - & 1.0 & - & - & - & - & - & 0.813953 & 0.794250 & \\
\hline - & 1.3 & - & - & - & - & - & 0.844088 & 0.868534 & \\
\hline- & 1.6 & - & - & - & - & - & 0.849653 & 0.924621 & \\
\hline- & - & 0.6 & - & - & - & - & 0.964351 & 0.897547 & \\
\hline- & - & 1.0 & - & - & - & - & 0.878902 & 0.917204 & \\
\hline- & - & 1.4 & - & - & - & - & 0.825589 & 0.931045 & \\
\hline- & - & - & 0.2 & - & - & - & 0.766833 & 0.873279 & \\
\hline- & - & - & 0.3 & - & - & - & 0.707801 & 0.821959 & \\
\hline- & - & - & 0.4 & - & - & - & 0.648491 & 0.776068 & \\
\hline- & - & - & - & 0.2 & - & - & 0.643005 & 0.772072 & \\
\hline- & - & - & - & 0.3 & - & - & 0.637516 & 0.768114 & \\
\hline- & - & - & - & 0.4 & - & - & 0.632025 & 0.764194 & \\
\hline- & - & - & - & - & 5.0 & - & 0.640043 & 0.750470 & \\
\hline- & - & - & - & - & 10 & - & 0.641015 & 0.747467 & \\
\hline- & - & - & - & - & $\infty$ & - & 0.641929 & 0.743954 & \\
\hline- & - & - & - & - & - & 0.8 & 0.732775 & 0.853349 & \\
\hline- & - & - & - & - & - & 1.1 & 0.814385 & 0.953775 & \\
\hline - & - & - & - & - & - & 1.4 & 0.889111 & 1.047541 & \\
\hline
\end{tabular}

\begin{tabular}{|c|c|c|c|c|c|c|c|c|c|}
\hline \multirow{2}{*}{$M$} & \multirow{2}{*}{$\operatorname{Pr}$} & \multirow{2}{*}{ Le } & \multirow{2}{*}{$E c_{x}$} & \multirow{2}{*}{$E c_{y}$} & \multirow{2}{*}{$\beta$} & \multirow{2}{*}{$S$} & \multicolumn{3}{|c|}{$\frac{S h_{x}}{\sqrt{R e_{x}}}=\frac{S h_{y}}{\sqrt{R e_{y}}}$} \\
\hline & & & & & & & PST case $\left[-\phi^{\prime}(0)\right]$ & PHF case [ & {$\left[\frac{1}{\phi(0)}\right]$} \\
\hline 0.5 & 1.2 & 1.2 & 0.1 & 0.1 & 1.0 & 0.5 & 1.279449 & 1.165181 & \\
\hline 1.0 & - & - & - & - & - & - & 1.245482 & 1.132613 & \\
\hline 1.5 & - & - & - & - & - & - & 1.207667 & 1.092575 & \\
\hline - & 1.0 & - & - & - & - & - & 1.032777 & 0.967284 & \\
\hline - & 1.3 & - & - & - & - & - & 1.293007 & 1.152761 & \\
\hline- & 1.6 & - & - & - & - & - & 1.540295 & 1.325481 & \\
\hline- & - & 0.6 & - & - & - & - & 0.728899 & 0.820085 & \\
\hline- & - & 1.0 & - & - & - & - & 1.306616 & 1.169738 & \\
\hline- & - & 1.4 & - & - & - & - & 1.759549 & 1.471945 & \\
\hline- & - & - & 0.2 & - & - & - & 1.793373 & 1.496479 & \\
\hline- & - & - & 0.3 & - & - & - & 1.836422 & 1.521693 & \\
\hline- & - & - & 0.4 & - & - & - & 1.879697 & 1.547613 & \\
\hline- & - & - & - & 0.2 & - & - & 1.883667 & 1.550003 & \\
\hline- & - & - & - & 0.3 & - & - & 1.887638 & 1.552399 & \\
\hline- & - & - & - & 0.4 & - & - & 1.891611 & 1.554802 & \\
\hline- & - & - & - & - & 5.0 & - & 1.779278 & 1.456543 & \\
\hline- & - & - & - & - & 10 & - & 1.758903 & 1.439395 & \\
\hline- & - & - & - & - & $\infty$ & - & 1.73627 & 1.420569 & \\
\hline- & - & - & - & - & - & 0.8 & 1.865901 & 1.549862 & \\
\hline - & - & - & - & - & - & 1.1 & 1.981162 & 1.658172 & \\
\hline - & _- & - & - & - & - & 1.4 & 2.086825 & 1.753786 & \\
\hline
\end{tabular}




\section{Conclusions}

In this article, MHD Casson nanofluid flow driven by an unsteady bidirectional moving surface subject to prescribed heat mechanism, namely, PST and PHF has been studied. The aspects of Brownian motion plus thermophoresis have been analyzed by using the mathematical relation of Buongiorno model. Similarity variables have been used to transform the governing PDEs into ODEs and then computational treatment has been made through KellerBox method. It is concluded through present study that PHF mechanism produces the better rate of heat transfer as compared to PST mechanism, whereas PST mechanism produces the better rate of mass transfer as compared to PHF mechanism. Moreover, it is also observed that the escalating amount of Eckert number enhances the rate of mass transfer, and reduces the rate of heat transfer.

Finally, it is hoped that the outcomes through present investigation will not be only helpful for industrial and engineering applications but also will be beneficiary for future endeavors.

Acknowledgements The authors are thankful to the mysterious reviewer(s) valuable comments that enriched the entire document.

\section{Compliance with ethical standards}

Conflict of interest The authors declare that they have no conflict of interest.

\section{References}

1. Crane LJ (1970) Flow past a stretching plate. Zeitschrift für angewandte Mathematik und Physik ZAMP 21(4):645-647

2. Chakrabarti A, Gupta AS (1979) Hydromagnetic flow and heat transfer over a stretching sheet. Q Appl Math 37(1):73-78

3. Hayat T, Javed T (2007) On analytic solution for generalized three-dimensional MHD flow over a porous stretching sheet. Phys Lett A 370(3-4):243-250

4. Liu IC, Andersson $\mathrm{HI}$ (2008) Heat transfer over a bidirectional stretching sheet with variable thermal conditions. Int J Heat Mass Transf 51(15-16):4018-4024

5. Elbashbeshy EMA, Bazid MAA (2004) Heat transfer over an unsteady stretching surface. Heat Mass Transf 41(1):1-4

6. Hsiao KL (2016) Stagnation electrical MHD nanofluid mixed convection with slip boundary on a stretching sheet. Appl Therm Eng 98:850-861

7. Raju CSK, Sandeep N (2016) Unsteady three-dimensional flow of Casson-Carreau fluids past a stretching surface. Alex Eng $J$ 55(2):1115-1126

8. Maity S, Singh SK, Kumar AV (2016) Unsteady three dimensional flow of Casson liquid film over a porous stretching sheet in the presence of uniform transverse magnetic field and suction/ injection. J Magn Magn Mater 419:292-300

9. Murthy MK (2018) MHD three dimensional flow of Casson fluid over an unsteady exponentially stretching sheet with slip conditions. In: Defect and diffusion forum, vol 388, pp 77-95. Trans Tech Publications Ltd

10. Hsiao KL (2017) Micropolar nanofluid flow with MHD and viscous dissipation effects towards a stretching sheet with multimedia feature. Int J Heat Mass Transf 112:983-990

11. Butt AS, Tufail MN, Ali A (2016) Three-dimensional flow of a magnetohydrodynamic Casson fluid over an unsteady stretching sheet embedded into a porous medium. J Appl Mech Tech Phys 57(2):283-292

12. Ahmad K, Nazar R (2011) Magnetohydrodynamic three-dimensional flow and heat transfer over a stretching surface in a viscoelastic fluid. J Sci Technol 3(1):1-10

13. Nadeem S, Haq RU, Akbar NS, Khan ZH (2013) MHD threedimensional Casson fluid flow past a porous linearly stretching sheet. Alex Eng J 52(4):577-582

14. Mahanta G, Shaw S (2015) 3D Casson fluid flow past a porous linearly stretching sheet with convective boundary condition. Alex Eng J 54(3):653-659

15. Buongiorno J (2006) Convective transport in nanofluids. J Heat Transf 128(3):240-250

16. Khan WA, Pop I (2010) Boundary-layer flow of a nanofluid past a stretching sheet. Int J Heat Mass Transf 53(11-12):2477-2483

17. Haq R, Nadeem S, Khan Z, Okedayo T (2014) Convective heat transfer and MHD effects on Casson nanofluid flow over a shrinking sheet. Open Phys 12(12):862-871

18. Nadeem S, Mehmood R, Akbar NS (2014) Optimized analytical solution for oblique flow of a Casson-nano fluid with convective boundary conditions. Int J Therm Sci 78:90-100

19. Nadeem S, Haq RU, Akbar NS (2013) MHD three-dimensional boundary layer flow of Casson nanofluid past a linearly stretching sheet with convective boundary condition. IEEE Trans Nanotechnol 13(1):109-115

20. Hayat T, Ashraf MB, Shehzad SA, Alsaedi A (2015) Mixed convection flow of Casson nanofluid over a stretching sheet with convectively heated chemical reaction and heat source/sink. J Appl Fluid Mech 8(4):2245-2256

21. Hussain T, Shehzad SA, Alsaedi A, Hayat T, Ramzan M (2015) Flow of Casson nanofluid with viscous dissipation and convective conditions: a mathematical model. J Cent South Univ 22(3):1132-1140

22. Mondal S, Oyelakin IS, Sibanda P (2017) Unsteady MHD threedimensional Casson nanofluid flow over a porous linear stretching sheet with slip condition. Front Heat Mass Transf (FHMT). https://doi.org/10.5098/hmt.8.37

23. Ibrar N, Reddy MG, Shehzad SA, Sreenivasulu P, Poornima T (2020) Interaction of single and multi-walls carbon nanotubes in magnetized-nano Casson fluid over radiated horizontal needle. SN Appl Sci 2(4):1-12

24. Hsiao KL (2017) To promote radiation electrical MHD activation energy thermal extrusion manufacturing system efficiency by using Carreau-nanofluid with parameters control method. Energy 130:486-499

25. Vo DD, Hedayat $M$, Ambreen T, Shehzad SA, Sheikholeslami M, Shafee A, Nguyen TK (2020) Effectiveness of various shapes of $\mathrm{Al}_{2} \mathrm{O}_{3}$ nanoparticles on the MHD convective heat transportation in porous medium. J Therm Anal Calorim 139(2):1345-1353

26. Sheikholeslami M, Rezaeianjouybari B, Darzi M, Shafee A, Li Z, Nguyen TK (2019) Application of nano-refrigerant for boiling heat transfer enhancement employing an experimental study. Int J Heat Mass Transf 141:974-980

27. Durgaprasad P, Varma SVK, Hoque MM, Raju CSK (2019) Combined effects of Brownian motion and thermophoresis parameters on three-dimensional (3D) Casson nanofluid flow across the porous layers slendering sheet in a suspension of graphene nanoparticles. Neural Comput Appl 31(10):6275-6286 
28. Chamkha AJ, Aly AM, Mansour MA (2010) Similarity solution for unsteady heat and mass transfer from a stretching surface embedded in a porous medium with suction/injection and chemical reaction effects. Chem Eng Commun 197(6):846-858

29. Pushpalatha K, Sugunamma V, Reddy JR, Sandeep N (2016) Heat and mass transfer in unsteady MHD Casson fluid flow with convective boundary conditions. Int J Adv Sci Technol 91:19-38

30. Ahmad I, Faisal M, Javed T (2019) Magneto-nanofluid flow due to bidirectional stretching surface in a porous medium. Spec Top Rev Porous Media Int J 10(5):457-473

31. Ahmad I, Faisal M, Javed T (2019) Bi-directional stretched nanofluid flow with Cattaneo-Christov double diffusion. Results Phys 15:102581

32. Ahmad I, Faisal M, Javed T (2020) Radiation aspects on magnetoCarreau nanoliquid flow over a bidirectionally stretchable surface with variable thermal conditions. Heat Transf. https://doi. org/10.1002/htj.21782

33. Faisal M, Ahmad I, Javed T (2020) Radiative nanofluid flow due to unsteady bi-directional stretching surface with convective and zero mass flux boundary conditions: using Keller box scheme. Comput Therm Sci. https://doi.org/10.1615/ComputThermalSc ien.2020033674

34. Javed T, Faisal M, Ahmad I (2020) Actions of viscous dissipation and Ohmic heating on bidirectional flow of a magneto-Prandtl nanofluid with prescribed heat and mass fluxes. Heat Transf. https://doi.org/10.1002/htj.21853

35. Javed T, Faisal M, Ahmad I (2020) Dynamisms of solar radiation and prescribed heat sources on bidirectional flow of magnetized Eyring-Powell nanofluid. Case Stud Therm Eng 21:100689

36. Faisal M, Ahmad I, Javed T (2020) Numerical simulation of mixed convective 3D flow of a chemically reactive nanofluid subject to convective Nield's conditions with the nonuniform heat source/ sink. Heat Transf 14:19. https://doi.org/10.1002/htj.21880

37. Keller HB (1978) Numerical methods in boundary-layer theory. Annu Rev Fluid Mech 10(1):417-433

Publisher's Note Springer Nature remains neutral with regard to jurisdictional claims in published maps and institutional affiliations. 